\title{
Intralobar Pulmonary Sequestration and Increased Serum CA 19-9
}

\author{
Massimo Montalto ${ }^{1}$, Michele Impagnatiello ${ }^{1}$, Maria Anna Nicolazzi ${ }^{1}$, Maria Teresa Congedo ${ }^{2}$, Raffaele Landolfi ${ }^{1}$ \\ ${ }^{1}$ Department of Internal Medicine, Gemelli Hospital, Catholic University, Rome, Italy \\ ${ }^{2}$ Department of Thoracic Surgery, Gemelli Hospital, Catholic University, Rome, Italy
}

How to cite this article: Montalto M, Impagnatiello MA, Nicolazzi M, Congedo MT, Landolfi R. Intralobar pulmonary sequestration and increased serum CA 19-9. EJCRIM 2017;4: doi:10.12890/2017_000583.

Conflicts of Interests: The Authors declare that there are no competing interests.

This article is licensed under a Commons Attribution Non-Commercial 4.0 License

\section{ABSTRACT}

Intralobar pulmonary sequestration is an uncommon congenital lung anomaly which consists of a mass of normal lung tissue not connected to the normal tracheobronchial tree and supplied by an anomalous systemic artery. Carbohydrate antigen 19-9 (CA 19-9) is widely accepted as a tumour marker for biliary, pancreatic and gastrointestinal cancer. However, CA 19-9 may also be increased in patients with benign disease. We describe the case of a 56-year-old woman with intralobar pulmonary sequestration who underwent unnecessary and extensive diagnostic abdominal examinations because of an increase in CA 19-9 serum levels.

\section{LEARNING POINTS}

- Knowledge of pulmonary sequestration causing increased serum CA 19-9 is important for the internist because it can help in the differential diagnosis even with neoplastic disease.

- Such awareness can also decrease the use of antibiotics.

- Familiarity with the condition can reduce the number of invasive examinations performed to exclude neoplasms of the gastrointestinal tract.

\section{KEYWORDS}

Pulmonary sequestration; CA 19-9

\section{INTRODUCTION}

Pulmonary sequestration (PS) is a rare congenital abnormality characterized by the presence of non-functioning lung tissue lacking communication with normal bronchial trees and supplied by an anomalous systemic artery instead of a pulmonary arterial branch ${ }^{[1,2]}$. There are two forms of PS: intralobar and extralobar. Both probably develop from an accessory lung bud in the primitive foregut. In the intralobar form, the sequestered section of the lung lies within the normal pulmonary visceral pleura, while in extralobar sequestration, the pulmonary tissue is separated from normal lung, is surrounded by its own pleura, and can also be extrathoracic ${ }^{[3,4]}$.

Since it is an anatomical condition, PS is usually associated with pulmonary infection, which is the most common clinical feature, although non-specific symptoms may also be present. PS may occasionally be misdiagnosed as a pulmonary abscess or tumour and only correctly identified after surgery ${ }^{[4]}$.

Carbohydrate 19-9 antigen (CA 19-9) is a glycoprotein mainly expressed on the cell surfaces of biliary and pancreatic duct cells. High CA 19-9 levels are considered strongly suggestive of malignancy of the pancreas, biliary system or gastrointestinal tract. However, slight increases in this marker (usually not more than four to five times the normal limit) are reported in several non-malignant diseases such as chronic hepatitis, primary biliary cirrhosis ${ }^{[5,6]}$ and primary sclerosing cholangitis ${ }^{[7]}$. An increase in CA $19-9$ has been rarely reported in some 
cases of $P S^{[2,3]}$.

We describe the case of a patient with recurrent pulmonary infections associated with an elevated serum CA 19-9 level, who underwent extensive gastrointestinal diagnostic evaluation before intralobar PS was identified.

\section{CASE REPORT}

A 56-year-old woman was admitted to Gemelli Hospital with asthenia and a 6-month history of fever and cough. Since symptoms persisted after antibiotic therapy (ciprofloxacin and clarithromycin) together with steroids (oral prednisone 25 mg daily for 2 weeks, with subsequent tapering over 3 weeks), a CT scan of the chest was performed 3 months later and showed multiple parenchymal consolidations in the basal lobe of the left lung and shaded 'ground glass' areas. The pneumologist therefore suggested another course of antibiotics (piperacillin/ tazobactam and trimethoprim). Since a lung neoplasm was suspected, the patient's general practitioner also ordered tests for tumour serological markers which showed increased CA 19-9. Consequently, to exclude an abdominal malignancy, the patient underwent gastroscopy, colonoscopy, a US scan and a CT scan, none of which revealed significant abnormalities.

A week after discontinuation of antibiotic therapy, the patient complained of a recurrence of fever and cough without sputum and attended our emergency room.

At admission in our department she was in good general condition with no pulmonary or digestive symptoms. No fever or other specific abnormalities were reported. The patient was a biologist, an ex-smoker and had no family history of pulmonary tuberculosis or bronchiectasis. Physical examination revealed only a minimal decrease in vesicular sounds in the left lower lung field.

Blood tests showed an increase in inflammatory markers (C-reactive protein $60.3 \mathrm{mg} / \mathrm{l}$, fibrinogen $647 \mathrm{mg} / \mathrm{dl}$ ), while procalcitonin was normal and beta-D-glucan was negative. Increased serum CA 19-9 levels were confirmed (240 IU/ml).

The CT scan showed a parenchymal consolidation with necrotic-like phenomena in the left lower lobe with inadequate recognition of subsegmental bronchial structures. The presence of an aberrant artery arising from the superior border of the aorta was suspected (Fig. 1).

In order to identify the aetiology of the infection, bronchoscopy with bronco-alveolar lavage (BAL) was performed. Endoscopic examination was negative as were microbiological tests (BAL and blood culture, Legionella urinary antigen test, pneumococcal urinary antigen test, Staphylococcus aureus nasal swab, throat swab, Quantiferon test, and serology for Mycoplasma pneumoniae, Chlamydia pneumoniae, and influenza A and B virus). The antistreptolysin O titre was normal.

Intravenous antibiotic therapy with meropenem $1 \mathrm{~g}$ three times a day and clindamycin $900 \mathrm{mg}$ three times a day was administered for 10 days during hospitalization and continued at home with oral clindamycin $300 \mathrm{mg}$ times a day and cefditoren $400 \mathrm{mg}$ twice a day. Because fever episodes and cough persisted over the following 2 weeks, the patient was readmitted and underwent surgery.

Surgery confirmed the presence of an arterial vessel from the abdominal aorta that supplied the left lower lung (Fig. 2). Histological examination of the resected tissue revealed chronic mucopurulent inflammation of the bronchial mucosa and lung tissue, as well as microabscess formation in the focal area, all findings which were consistent with PS. After pulmonary resection, fever, inflammatory blood markers and serum CA 199 levels (Fig. 3) rapidly decreased to normal ranges.

The patient is well 6 months after surgery with no recurrence of symptoms.

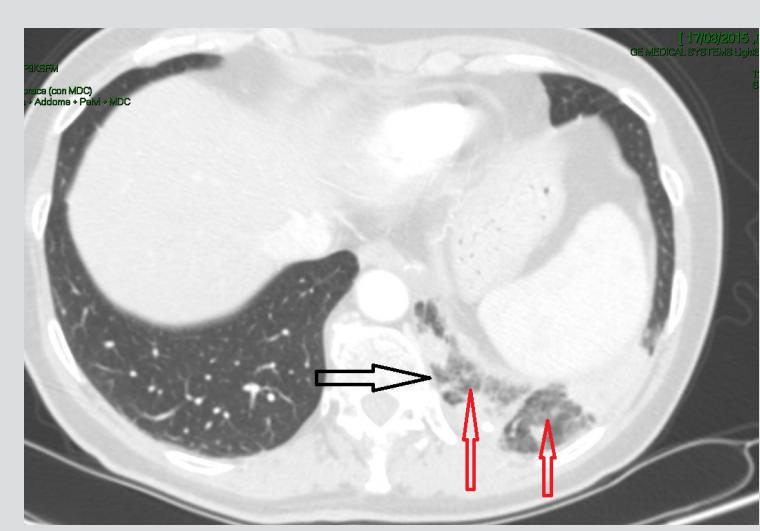

Figure 1. Chest CT showing a heterogeneous low density mass located in the left lower lobe (red arrows) and an aberrant artery (black arrow)

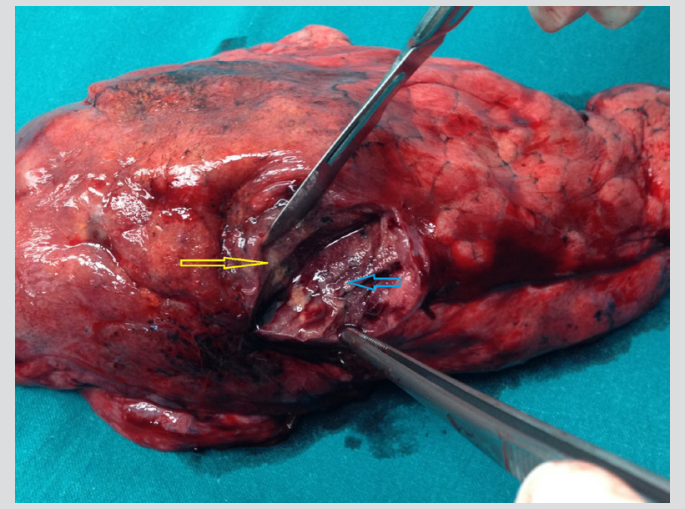

Figure 2. Left lower lobe: dilated bronchioles with a thickened wall (blue arrow) and small cavities and multiple abscesses containing necrotic debris, stagnant blood or fluid (yellow arrow) 


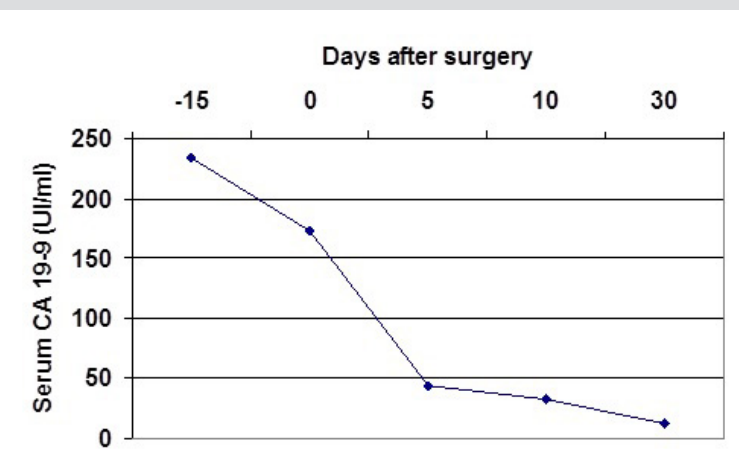

Figure 3. Serum carbohydrate antigen 19-9 (CA19-9) levels before and after surgery for intralobar pulmonary sequestration

\section{DISCUSSION}

To best of our knowledge, this is the first report from Italy of PS associated with an increase in CA 19-9. PS is a rare lung congenital malformation where the absence of a connection with the normal bronchial tree favours infection and can be a cause of recurrent pneumonia. The most common clinical features are fever and cough due to various bacteria and occasionally uncommon pathogens such as Mycobacterium tuberculosis and fungus ${ }^{[1-4]}$.

The presence of high levels of CA 19-9 is usually considered to strongly suggest malignant pancreatic-biliary or gastrointestinal disease, but this serum marker may also increase in a number of non-malignant diseases ${ }^{[5]}$. In patients with PS, an increase in serum CA 19.9 has been reported anecdotally ${ }^{[3-6]}$. Only a few cases of elevated CA 19-9 serum values in association with PS have been reported to date ${ }^{[6]}$.

Clinicians are not generally aware of the relationship between elevated CA 19.9 levels and PS because the condition is rare and also likely underestimated. Indeed, when recurrent pneumonia is seen in clinical practice, CA 19-9 tests are not requested by physicians if symptoms of malignancy are absent. Furthermore, when an increase in CA 19-9 has been reported in patients with PS, as in our case, no abdominal symptoms are found and in most cases it is an occasional finding ${ }^{[2,3]}$.

CA 19-9 is synthesized by normal bronchial epithelial cells, and is elevated in blood during epithelial cell proliferation and accumulation in PS tissue. Other reports indicate that proliferation of bronchial epithelial cells causes CA 19-9 elevation via a chronic inflammatory process due to infection by pathogenic organisms such as Aspergillus or Mycobacterium.

Our case report highlights two points. The anatomic lung anomaly was not promptly found at the first outpatient CT scan although postdiagnostic review of images showed radiological features suggestive of PS were already present. Therefore, in patients with a history of recurrent pulmonary infection, PS should be considered a cause of poor image resolution, even though it is a rare pathological condition. Second, we underline the difficulty in identifying the cause of an increase in CA 19-9 when PS is not recognized. In such cases patients may undergo a large number of unnecessary diagnostic examinations.

In conclusion, our case confirms the association between PS and increased CA 19-9 levels. Consequently, in patients with PS and elevated CA 19-9, an extensive diagnostic process aimed at excluding abdominal malignancy is not necessary. Further basic studies regarding the mechanism of CA 19-9 increase in PS are required.

\section{REFERENCES}

1. Savic B, Birtel FJ, Tholen W, Funke HD, Knoche R. Lung sequestration: report of seven cases and review of 540 published cases. Thorax 1979;34:96-101.

2. Ahn YH, Song MJ, Park SH. Intralobar pulmonary sequestration showing increased serum CA19-9. Tuberc Respir Dis 2012;72:507-510.

3. Armbruster C, Kriwanek S, Feichtinger H, Armbruster C. Intra-abdominal sequestration of the lung and elevated serum levels of CA 19-9: a diagnostic pitfall. HPB (Oxford) 2004;6:45-48.

4. Kristensen KL, Duus LA, Elle B. Endovascular treatment of an aneurysmal aberrant systemic artery supplying a pulmonary sequestrum. Cardiovasc Intervent Radiol 2015;38:1308-1311.

5. Gupta MK, Arciaga R, Bocci L, Tubbs R, Bukowski R, Deodhar SD. Measurement of a monoclonal-antibody- defined antigen (CA19-9) in the sera of patients with malignant and nonmalignant diseases: comparison with carcinoembryonic antigen. Cancer 1985;56:277-283.

6. Dong J, Cai Y, Chen R, Du S, Chen Y, Shi K. A case report and a short literature review of pulmonary sequestration showing elevated serum levels of carbohydrate antigen 19-9. J Nippon Med Sch 2015;82:211-215.

7. Montalto M, Santoro L, Vastola M, Curigliano V, Ricci R, Vecchio FM, et al. Normalisation of high CA 19-9 values in autoimmune hepatitis after steroidal treatment. Int J Immunopathol Pharmacol 2005;18:603-607. 